\title{
Structured interdisciplinary bedside rounds, in-hospital deaths, and new nursing home placements among older inpatients
}

This article was published in the following Dove Press journal:

Clinical Interventions in Aging

\author{
David Basic' \\ Elizabeth Huynh ${ }^{2}$ \\ Rinaldo Gonzales' \\ Chris Shanley ${ }^{3}$ \\ 'Department of Geriatric Medicine, \\ Aged Care Research Unit, Liverpool \\ Hospital, Liverpool BC, NSW I87I, \\ Australia; ${ }^{2}$ Department of Geriatric \\ Medicine, Aged Care Research Unit, \\ Liverpool Hospital, University of \\ NSW, Liverpool BC, NSW I87I, \\ Australia; ${ }^{3}$ Department of Geriatric \\ Medicine, Centre for Applied \\ Nursing Research, Western Sydney \\ University and Ingham Institute of \\ Applied Medical Research, Aged Care \\ Research Unit, Liverpool Hospital, \\ Liverpool BC, NSW I87I, Australia
}

Correspondence: David Basic Department of Geriatric Medicine, Liverpool Hospital, Locked Mail Bag 7I03, Liverpool BC, New South Wales |87|, Australia

Tel +61287386152

Fax +6I 298286114

Email david.basic@health.nsw.gov.au
Purpose: Ineffective interdisciplinary communication is linked to many adverse consequences of hospitalization. This study evaluated the effect of SIBR, a model of care that encourages interdisciplinary communication and patient and family participations, on in-hospital deaths and new nursing home $(\mathrm{NH})$ placements.

Materials and methods: This before-after study included 3,673 consecutive inpatients of mean age 83.8 years, of whom $93.2 \%$ were admitted through the emergency department. After each twice-weekly SIBR session, an interdisciplinary care plan was implemented and recorded on a datasheet attached to the bedside record. Staff unable to participate in SIBR were asked to view the datasheet and to follow the care plan. Logistic regression models were computed for in-hospital deaths and new NH placements.

Results: Although SIBR implementation had no effect on in-hospital deaths (OR, 1.00; 95\% CI, 0.77-1.29), SIBR increased NH placements among those who survived the hospitalization $(\mathrm{n}=3,346)$ in both unadjusted $(14.6 \%$ vs $9.1 \% ; P<0.001)$ and adjusted $(\mathrm{OR}, 1.75 ; 95 \% \mathrm{CI}$, 1.38-2.23) analyses.

Conclusion: Although the mechanisms between SIBR implementation and NH placement remain uncertain, SIBR may encourage patients and families to make decisions on placement earlier than they would have otherwise. Models of care aiming to improve communication should be evaluated across diverse services and settings to determine effectiveness and to monitor for adverse findings.

Keywords: aged, communication, death, inpatients, nursing homes, safety

\section{Introduction}

The adverse consequences of hospitalization, such as medication errors, ${ }^{1}$ hospitalacquired infections, ${ }^{2}$ preventable injuries, and preventable deaths, ${ }^{3}$ have encouraged the implementation of new models of interdisciplinary care that aim to address these issues by improving cooperation and communication among health care professionals. However, interdisciplinary care may be sporadic, unstructured, and geographically fragmented, ${ }^{4}$ or it may lack physician's involvement and leadership ${ }^{5}$ or structured communication protocols. ${ }^{6,7}$

The use of structured interdisciplinary bedside rounds (SIBRs) is a new model of care that brings all members of the interdisciplinary team to the patient's bedside, thus promoting interprofessional accountability and encouraging the patient and the family to participate in most aspects of care. ${ }^{8}$ The use of a standardized communication protocol with a safety checklist (Table 1) reflects best practice in communication. ${ }^{8}$ 
Table I SIBR communication protocol

\begin{tabular}{|c|c|}
\hline Information exchanged & $\begin{array}{l}\text { Duration } \\
\text { (seconds) }\end{array}$ \\
\hline $\begin{array}{l}\text { Introduction (senior doctor or NUM) } \\
\text { Greet patient and family, introduce team }\end{array}$ & 15 \\
\hline $\begin{array}{l}\text { Medical (senior doctor) } \\
\text { Premorbid domicile and reason for presentation } \\
\text { Active diagnoses and response to treatment }{ }^{\mathrm{a}} \\
\text { Tests, procedures, and consultant inputs yet } \\
\text { to be performed }\end{array}$ & 45 \\
\hline $\begin{array}{l}\text { Nursing (bedside nurse) } \\
\text { Relevant events during previous } 48 \text { hours, } \\
\text { including vital signs and MET calls } \\
\text { Documentation of resuscitation status } \\
\text { Concerns related to food and fluid intake, } \\
\text { bladder, and bowel output } \\
\text { Bladder and intravenous catheterization } \\
\text { Safety checklist } \\
\text { Behavior, including aggression and attempts } \\
\text { to abscond } \\
\text { Pressure care } \\
\text { Falls }\end{array}$ & 60 \\
\hline $\begin{array}{l}\text { Allied health update and plan } \\
\text { Physiotherapist } \\
\text { Occupational therapist } \\
\text { Social worker } \\
\text { Speech pathologist } \\
\text { Dietician } \\
\text { Neuropsychologist }\end{array}$ & 60 \\
\hline $\begin{array}{l}\text { Patient and family } \\
\text { Add information, correct misinformation, } \\
\text { invite limited questions }{ }^{c}\end{array}$ & 45 \\
\hline $\begin{array}{l}\text { Summary (consultant and/or team registrar) } \\
\text { Verbalize care plan, including EDD and } \\
\text { discharge domicile }\end{array}$ & 15 \\
\hline
\end{tabular}

Notes: ${ }^{2}$ Active diagnoses (acute and chronic) were those that affected physical, social, or psychological function, or those that needed medication changes, investigations, or increased monitoring. 'Delays were identified and escalated as appropriate by a senior nurse or doctor. 'Although patient and family inputs were addressed at any time during the communication protocol, prolonged discussion was deferred until the completion of the SIBR.

Abbreviations: EDD, estimated date of discharge; MET, medical emergency team; NUM, nurse unit manager; SIBR, structured interdisciplinary bedside round.

Whether SIBR is effective remains inconclusive. Stein et $\mathrm{al}^{8}$ reported that daily SIBR reduced the unadjusted length of stay (LOS) and in-hospital deaths among medical inpatients, while Huynh et $\mathrm{al}^{9}$ found that twice-weekly SIBR failed to reduce LOS or 28-day readmission rates among older inpatients. No data are available on the effect of SIBR on new nursing home (NH) placement.

Despite the scarcity of evidence of its effectiveness, SIBR has been widely implemented across diverse services in many hospitals in Australia, Canada, and USA, and a similar model has been endorsed by the Royal College of Physicians and the Royal College of Nursing in the UK. ${ }^{10}$

In this article, we evaluate the associations between SIBR and in-hospital death and new NH placement.
We hypothesized that SIBR would reduce both outcomes by improving interdisciplinary communication, incorporating a safety checklist, and enabling patients and family members to correct misinformation and to formulate a management plan.

\section{Materials and methods Study location and study patients}

This before-after study of consecutive patients admitted to an aged care service at a tertiary hospital in Sydney, Australia, was conducted between January 1, 2013 and September 30, 2015. The number of patients in the control (before) group and the intervention (after) group were 1,703 and 1,970, respectively. In a 2-month hiatus (May 1 to June 30, 2014) between the before and after study arms, aged care staff completed SIBR training. The study was approved by the South Western Sydney Local Health District Human Research Ethics Committee (Approval no: 14/334 LNR). Patient consent was not required because the study was deemed to be of low and negligible risk.

\section{Intervention and other aged care practices}

The twice-weekly SIBR communication protocol is shown in detail in Table 1. After each 3- to 5-minute interaction, a care plan was verbalized by a senior doctor and documented on a datasheet. This was attached to the bedside notes to allow staff unable to participate in SIBR to understand and implement the care plan. Attendance by family and carers was encouraged.

Except for weekly case conferences, which were shorter during SIBR, all other practices were similar before and during SIBR. During SIBR, case conferences were limited to up to 10 patients, to minimize repetition of material already discussed but to allow detailed discussion of complex patients and of sensitive information (eg, elder abuse).

\section{Measures}

Data that were collected before and during SIBR included demographics, admission and discharge domicile, referral source, frailty (present 1 month before admission and measured using all available sources), active diagnoses, in-hospital deaths, and new NH placements. The degree of frailty was determined using the Canadian Study of Health and Aging Clinical Frailty Scale (CSHA-CFS). ${ }^{11}$ Version 5.1 of the Australian Refined Diagnosis Related Groups classification system ${ }^{12}$ was used to assign active medical diagnoses. Active diagnoses, both acute and chronic, were those that affected physical, social, or psychological function, or those that needed medication changes, investigations, or increased 
monitoring to treat symptoms and direct management. To streamline care and discharge planning, tests, procedures and consultant inputs yet to be performed were monitored during SIBR and escalated when appropriate by a senior doctor or senior nurse (mostly nurse unit manager). Other measures documented during SIBR included patient and family participation rates, resuscitation status, and whether a medical emergency team call was initiated (Table 1).

\section{Statistical analyses}

Separate logistic regression models were computed for in-hospital deaths and new NH placements. Variables were considered for inclusion in the models based on the literature, ${ }^{13,14}$ biological credibility, effect modification (ie, whether the effect of SIBR was modified by English-speaking ability or the presence of dementia and/or delirium), statistical significance, and confounding. Comparisons between groups were tested using chi-squared tests for dichotomous variables, Kruskal-Wallis tests for ordinal variables, and 2 -sample $t$-tests for normally distributed variables. The study was powered to detect a $25 \%$ decrease in deaths and new $\mathrm{NH}$ placements (10\%-7.5\%; Type 1 error, 0.05; Type 2 error, 0.20). Analyses were performed using SAS version 9.4 (SAS Institute Inc., Cary, NC, USA).

\section{Results \\ Patient's characteristics and participation rates}

The mean age of all 3,673 patients was $83.8 \pm 7.7$ years, and $41.6 \%$ were male. Most (93.2\%) of the patients were admitted via the emergency department. Of the $58.6 \%$ born in non-English-speaking background (NESB) countries, 58.0\% were unable to speak English. Dementia and delirium were present in $46.6 \%$ and $45.9 \%$, respectively. Table 2 shows the characteristics of the participants before and during SIBR.

During 4,508 documented SIBR sessions (data recorded for up to eight sessions), 2,964 (65.7\%) patients participated. Despite encouragement, a family member was present in only 913 (20.3\%) documented sessions.

\section{SIBR, in-hospital deaths, and new $\mathrm{NH}$ placements}

The overall rates of death and new NH placement were $8.9 \%$ and $12.0 \%$, respectively. The implementation of SIBR did not reduce unadjusted deaths $(8.5 \%$ vs $9.2 \%$ before and during SIBR, respectively; $P=0.45)$. Similarly, SIBR had no effect on deaths in adjusted analysis (OR, 1.00; 95\% CI, 0.77-1.29; Table 3). Of those who survived the hospitalization ( $n=3346)$, SIBR implementation increased NH placements, in both

Table 2 Characteristics of study participants before and during implementation of SIBRs

\begin{tabular}{|c|c|c|c|c|c|}
\hline \multirow[t]{2}{*}{ Characteristic } & \multicolumn{2}{|l|}{ SIBR period } & \multirow[t]{2}{*}{ Characteristic } & \multicolumn{2}{|c|}{ SIBR period } \\
\hline & $\begin{array}{l}\text { Before } \\
(n=I, 703)\end{array}$ & $\begin{array}{l}\text { During } \\
(n=I, 970)\end{array}$ & & $\begin{array}{l}\text { Before } \\
(n=I, 703)\end{array}$ & $\begin{array}{l}\text { During } \\
(n=I, 970)\end{array}$ \\
\hline Age (years) & $83.8 \pm 7.5$ & $83.9 \pm 7.9$ & Medical diagnosis, n (\%) ${ }^{\mathrm{a}, \mathrm{b}}$ & & \\
\hline Male gender, n (\%) & $691(40.6)$ & $835(42.4)$ & Dementia & $757(50.8)$ & $744(43.0)$ \\
\hline NESB country of birth, $n(\%)$ & $994(58.4)$ & $\mathrm{I}, \mathrm{I} 60(58.9)$ & Delirium & $692(46.4)$ & $786(45.5)$ \\
\hline English speaking, n (\%) & $\mathrm{I}, \mathrm{I} 38$ (66.8) & $\mathrm{I}, 286(65.3)$ & Deconditioning & $418(28.1)$ & $489(28.3)$ \\
\hline Preadmission residence, $\mathrm{n}(\%)$ & & & Malnutrition (severe) & I 70 ( $\mid$ I.4) & $229(13.2)$ \\
\hline Home & $\mathrm{I}, \mathrm{I} 32(66.5)$ & $1,362(69.1)$ & Cardiac failure & $279(18.7)$ & $313(18.1)$ \\
\hline Low-level residential care & $160(9.4)$ & $81(4.1)$ & Acute renal failure & $303(20.3)$ & $372(21.5)$ \\
\hline High-level residential care & $4 I I(24.1)$ & $527(26.8)$ & COPD & $150(10.1)$ & $200(11.6)$ \\
\hline Referral source, n (\%) & & & Type 2 respiratory failure & $45(3.0)$ & $57(3.3)$ \\
\hline Emergency department & I,597 (93.8) & $\mathrm{I}, 827(92.7)$ & PTE & $27(1.8)$ & $35(2.0)$ \\
\hline Consult and transfer care & $104(6.1)$ & $138(7.0)$ & Stroke & $112(7.5)$ & $124(7.2)$ \\
\hline Others & $2(0.1)$ & $5(0.3)$ & Fracture (any) & I7| (II.5) & $223(12.9)$ \\
\hline CSHA-CFS category, n (\%) & & & Fracture pelvis & $33(2.2)$ & $50(2.9)$ \\
\hline 1 & I $(0.1)$ & $2(0.1)$ & Fracture vertebra $^{d}$ & $49(3.3)$ & $58(3.4)$ \\
\hline 2 & $3(0.2)$ & $3(0.2)$ & Fracture rib $^{d}$ & $28(1.9)$ & $50(2.9)$ \\
\hline 3 & $9(0.6)$ & $24(1.4)$ & Infection (any) & $876(58.8)$ & $I, 06 I(6 I .4)$ \\
\hline 4 & $61(4.1)$ & $73(4.2)$ & Infection respiratory tract & $422(28.3)$ & $567(32.8)$ \\
\hline 5 & $324(21.9)$ & 397 (22.7) & Septic shock & $88(5.9)$ & $140(8.1)$ \\
\hline 6 & $670(45.3)$ & $769(44.0)$ & Malignant neoplasm (any) & $200(13.4)$ & $153(8.9)$ \\
\hline 7 & $412(27.8)$ & $478(27.4)$ & Major depression & $4 \mid(2.8)$ & $45(2.6)$ \\
\hline
\end{tabular}

Notes: aMedical diagnosis data were missing for 2 I3 (I2.5\%) before SIBR and for 24 I (I2.2\%) during SIBR. ${ }^{b}$ Numbers and percentages for medical diagnoses and CSHA-CFS categories refer to patients with non-missing data. ${ }^{~ C S H A-C F S ~ c a t e g o r y ~ d a t a ~ w e r e ~ m i s s i n g ~ f o r ~} 223$ (I3.1\%) before SIBR and for 224 (II.4\%) during SIBR. ${ }^{\mathrm{d} F r a c t u r e}$ vertebra and fracture rib include both single and multiple fractures.

Abbreviations: CSHA-CFS, Canadian Study of Health and Aging Clinical Frailty Scale; NESB, non-English-speaking background; PTE, pulmonary thromboembolism; SIBR, structured interdisciplinary bedside round. 
Table 3 Logistic regression models for in-hospital deaths and new $\mathrm{NH}$ placements

\begin{tabular}{|c|c|c|}
\hline \multirow[t]{2}{*}{ Predictor } & $\begin{array}{l}\text { In-hospital } \\
\text { deaths }\end{array}$ & $\begin{array}{l}\text { New NH } \\
\text { placements }^{\mathrm{b}}\end{array}$ \\
\hline & OR $(95 \% \mathrm{Cl})$ & OR $(95 \% \mathrm{Cl})$ \\
\hline SIBR implementation & $\mathrm{I} .00(0.77-1.29)$ & $1.75(1.38-2.23)$ \\
\hline Age (years) & $1.03(1.01-1.05)$ & $0.99(0.98-1.01)$ \\
\hline CSHA-CFS & $2.28(1.89-2.77)$ & $1.27(1.09-1.48)$ \\
\hline Cardiac failure & $1.76(1.32-2.36)$ & \\
\hline Respiratory infection & $2.54(1.96-3.30)$ & \\
\hline Septic shock & $5.36(3.83-7.49)$ & \\
\hline Acute renal failure & $2.04(1.56-2.68)$ & \\
\hline Dementia & & $1.75(1.37-2.25)$ \\
\hline Deconditioning & & $1.77(1.39-2.25)$ \\
\hline Malnutrition (severe) & & I.7I (1.25-2.34) \\
\hline
\end{tabular}

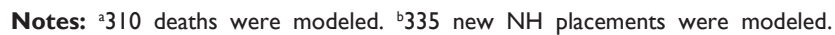
CSHA-CFS category data were missing for 223 (13.1\%) patients before SIBR and for 224 (II.4\%) patients during SIBR. Medical diagnostic data were missing for 213 (12.5\%) patients before SIBR and for 24I (12.2\%) patients during SIBR.

Abbreviations: CSHA-CFS, Canadian Study of Health and Aging Clinical Frailty Scale; $\mathrm{NH}$, nursing home; SIBR, structured interdisciplinary bedside round.

unadjusted ( $14.6 \%$ vs $9.1 \% ; P<0.001)$ and adjusted analyses (OR, 1.75; 95\% CI, 1.38-2.23; Table 3). The impact of SIBR on NH placement seemed to be higher in NESB patients (14.1\% [during] vs $7.5 \%$ [before]; $P<0.001)$ than in others $(15.3 \%$ vs $11.2 \% ; P=0.03)$. Patients born in NESB countries had similar characteristics before and during SIBR, except for a lower prevalence of dementia during SIBR (46.8\% vs $53.8 \%)$.

For both deaths and new NH placements, the effect of SIBR was not modified by English-speaking ability or the presence of dementia and/or delirium (all $P>0.05$ ).

\section{Missing data}

Medical diagnosis data were missing for 213 (12.5\%) patients before SIBR and for 241 (12.2\%) during SIBR. Frailty data (CSHA-CFS) were missing for 223 (13.1\%) patients before SIBR and for 224 (11.4\%) during SIBR. All other data were complete. Those with missing data were marginally younger ( 83.1 vs 83.9 years; $P=0.04$ ) and less likely to present via the emergency department $(88.8 \%$ vs $93.9 \% ; P<0.001)$ or to die in hospital $(3.5 \%$ vs $9.7 \% ; P<0.001)$. They also had a shorter median LOS ( 7 vs 8 days; $P=0.001$ ). All other characteristics, including the rates of $\mathrm{NH}$ placement, were similar.

\section{Discussion}

The use of SIBR is being widely implemented in Australia and in other countries despite the scarcity of peer-reviewed evidence. Although miscommunication is a leading cause of hospitalrelated errors, ${ }^{3}$ models of care aiming to improve communication may not be effective across all services and settings.
This large before-after study found that SIBR implementation increased NH placements among those who survived the hospitalization in both unadjusted and adjusted analyses. The implementation of SIBR had no effect on in-hospital deaths.

In Australia, most older people discharged from hospital to a NH stay there permanently. For the most part, this is an undesirable outcome, with many people reporting feelings of vulnerability; boredom and loneliness, ${ }^{15}$ and concerns about lack of staffing, privacy, space, autonomy, and dignity. ${ }^{16}$ These feelings may be of a greater concern to $\mathrm{NH}$ residents than challenges due to physical or functional disability. ${ }^{15} \mathrm{NH}$ placement is expensive and is associated with increased mortality. ${ }^{17}$

The requirement to discuss the discharge domicile with patients and families during SIBR may have streamlined the placement process and appropriately increased $\mathrm{NH}$ placements. However, it is possible that SIBR encouraged patients and families to make decisions on placement earlier than they would have otherwise, particularly as refusals may have been more difficult when NH placement was recommended by a large interdisciplinary team. Due to an increasing demand for hospital beds, early decisions on discharge dates and domiciliary arrangements are encouraged to support patient flow. Thus, it is conceivable that the SIBR team recommended the discharge domicile earlier than previously, perhaps before patients recovered sufficiently from their illnesses.

Although we remain uncertain why SIBR implementation increased NH placements, of concern is our finding that NESB patients were more likely to be placed despite a lower prevalence of dementia (during SIBR). Limited data suggest that people from NESB countries are more likely to utilize community-based services in preference to $\mathrm{NH}$ placement. ${ }^{18,19}$

In contrast to the data published by Stein et al, ${ }^{8}$ SIBR implementation did not reduce in-hospital deaths. Although it is possible that daily SIBR (utilized by Stein et al) is more effective than twice-weekly SIBR (utilized by us), we believe that our alternative communication methods compensated for less frequent SIBR. These included systematic documentation of SIBR data on a datasheet (allowing all staff to know the care plan) and continuation of other practices during SIBR, such as weekday 30- to 45-minute journey board rounds and weekly case conferences (albeit shortened). Although these practices may explain our negative results, low family participation rates during SIBR may have reduced their benefit, particularly as many NESB patients were unable to speak English. Furthermore, a substantial proportion of participants had cognitive impairment, limiting their participation during SIBR. However, the effect of SIBR was not modified by English-speaking 
ability or the presence of cognitive impairment. It is also possible that 3- to 5-minute SIBR interactions are too brief to meaningfully affect death rates. Nonetheless, Stein et $\mathrm{al}^{8}$ utilized SIBR interactions of similar or shorter durations and yet reported a $50 \%$ reduction in deaths.

SIBR may reduce deaths in other patient groups and settings because the relationship between SIBR and death is affected by many variables. These include the effect of specific illnesses, the number of medical comorbidities, patient race and ethnicity (eg, being black or Hispanic in USA), racial and ethnic variations in treatment preferences, and the hospital care intensity of the participants' geographic region. ${ }^{20,21}$

Although the focus of our study was on the effect of SIBR on specific quantitative outcomes, SIBR implementation has been reported to improve qualitative outcomes such as staff perceptions of teamwork, communication, understanding of the care plan, and job satisfaction. ${ }^{22}$ While data are limited, Cao et $\mathrm{a}^{23}$ found a non-significant trend toward higher patient/family satisfaction in a study of SIBR in a medical intensive care unit.

The main limitations of our study are its design (beforeafter study rather than randomized controlled study) and its generalizability (single-hospital study). Also, 13.1\% of the patients had missing diagnostic or frailty data. Although all other data were complete, missing data may have affected the quantitative values shown in the logistic regression models (Table 3). However, we believe that our qualitative conclusions are valid. We also treated CSHA-CFS as a continuous variable, rather than as a categorical variable. Other authors have suggested a similar approach to reduce the number of terms in regression models. ${ }^{24}$ Despite these limitations, our study has several strengths. The study population was large, and we evaluated consecutive patients. We adjusted for key variables and included a measure of frailty (CSHA-CFS), the nature of whose items (a mixture of comorbidity, cognitive impairment, and disability) may have encapsulated unmeasured variables that affected deaths and NH placements.

\section{Conclusion}

SIBR implementation did not reduce in-hospital deaths and new NH placements among older medical inpatients. Of concern is our unexpected finding that SIBR implementation increased NH placements.

\section{Acknowledgments}

The authors acknowledge the following geriatricians for their contributions to data collection: Benyamen Benyamen, Leemin Chan, David Conforti, Angela Khoo, Florence Loh, Colin
Macarthur, Nyoka Ruberu, and Nalini Thayaparan. The authors also acknowledge the community volunteers (for their assistance during the training period), the project leads from the $\mathrm{CEC}$, and all staff on the aged care wards for helping to develop the communication protocol and implementing SIBR.

\section{Disclosure}

The authors report no conflicts of interest in this work.

\section{References}

1. Manojlovich M, DeCicco B. Healthy work environments, nurse-physician communication, and patients' outcomes. Am J Crit Care. 2007;16(6): 536-543.

2. Boev C, Xia Y. Nurse-physician collaboration and hospital-acquired infections in critical care. Crit Care Nurse. 2015;35(2):66-72.

3. McComb SA, Henneman EA, Hinchey KT, et al. Improving teamwork on general medical units: when teams do not work face-to-face. Jt Comm J Qual Patient Saf. 2012;38(10):471-478.

4. O’Leary KJ, Boudreau YN, Creden AJ, Slade ME, Williams MV. Assessment of teamwork during structured interdisciplinary rounds on medical units. J Hosp Med. 2012;7(9):679-683.

5. Begue A, Overcash J, Lewis R, et al. Retrospective study of multidisciplinary rounding on a thoracic surgical oncology unit. Clin J Oncol Nurs. 2012;16(6):E198-E202.

6. Curley C, McEachern JE, Speroff T. A firm trial of interdisciplinary rounds on the inpatient medical wards: an intervention designed using continuous quality improvement. Med Care. 1998;36(8 Suppl): AS4-AS12.

7. Dutton RP, Cooper C, Jones A, Leone S, Kramer ME, Scalea TM. Daily multidisciplinary rounds shorten length of stay for trauma patients. J Trauma. 2003;55(5):913-919.

8. Stein J, Payne C, Methvin A, et al. Reorganizing a hospital ward as an accountable care unit. J Hosp Med. 2015;10(1):36-40.

9. Huynh E, Basic D, Gonzales R, Shanley C. Structured interdisciplinary bedside rounds do not reduce length of hospital stay and 28-day re-admission rate among older people hospitalised with acute illness: an Australian study. Aust Health Rev. 2017;41(6):599-605.

10. Royal College of Physicians [webpage on the Internet]. Ward Rounds in Medicine: Principles for Best Practice. Available from: https://www. rcplondon.ac.uk/projects/outputs/ward-rounds-medicine-principlesbest-practice. Accessed April 19, 2018.

11. Rockwood K, Song X, MacKnight C, et al. A global clinical measure of fitness and frailty in elderly people. CMAJ. 2005;173(5): 489-495.

12. Australian Refined Diagnosis Related Groups. Hospital Casemix Protocol 2008-2009. Available from: https:/health.gov.au/internet/ main/publishing.nsf/Content/647C11F217FEDC1FCA257BF0001 ED9AF/\$File/HCP\%20Annual\%20Report\%200809.pdf. Accessed April 19, 2018.

13. Campbell SE, Seymour DG, Primrose WR; ACMEPLUS Project. A systematic literature review of factors affecting outcome in older medical patients admitted to hospital. Age Ageing. 2004;33(2):110-115.

14. Luppa M, Luck T, Weyerer S, König HH, Brähler E, Riedel-Heller SG. Prediction of institutionalization in the elderly. A systematic review. Age Ageing. 2010;39(1):31-38.

15. Slama CA, Bergman-Evans B. A troubling triangle. An exploration of loneliness, helplessness, and boredom of residents of a veterans home. J Psychosoc Nurs Ment Health Serv. 2000;38(12):36-43.

16. Duffin C. CQC criticises lack of privacy and dignity in England's care homes. Nurs Stand. 2013;27(29):10.

17. Wolinsky FD, Callahan CM, Fitzgerald JF, Johnson RJ. The risk of nursing home placement and subsequent death among older adults. J Gerontol. 1992;47(4):S173-S182. 
18. Runge C, Gillham J, Peut A [webpage on the Internet]. Transitions in Care of People With Dementia: A Systematic Review of the Literature; February 28, 2009. Available from: https://www.aihw.gov.au/reports/ aged-care/transitions-care-people-dementia-review/contents/table-ofcontents. Accessed April 19, 2018.

19. Mold F, Fitzpatrick JM, Roberts JD. Minority ethnic elders in care homes: a review of the literature. Age Ageing. 2005;34(2):107-113.

20. Kelley AS, Ettner SL, Wenger NS, Sarkisian CA. Determinants of death in the hospital among older adults. J Am Geriatr Soc. 2011;59(12): 2321-2325.

21. Barnato AE, Anthony DL, Skinner J, Gallagher PM, Fisher ES. Racial and ethnic differences in preferences for end-of-life treatment. J Gen Intern Med. 2009;24(6):695-701.
22. Gausvik C, Lautar A, Miller L, Pallerla H, Schlaudecker J. Structured nursing communication on interdisciplinary acute care teams improves perceptions of safety, efficiency, understanding of care plan and teamwork as well as job satisfaction. J Multidiscip Healthc. 2015;8: 33-37.

23. Cao V, Tan LD, Horn F, et al. Patient-centered structured interdisciplinary bedside rounds in the medical ICU. Crit Care Med. 2018;46(1): 85-92.

24. Kleinbaum DG, Kupper LL, Morgenstern H. Modeling: Analysis Strategy. Epidemiologic Research, Principles and Quantitiative Methods. New York: John Wiley \& Sons; 1982:447-456.
Clinical Interventions in Aging

\section{Publish your work in this journal}

Clinical Interventions in Aging is an international, peer-reviewed journal focusing on evidence-based reports on the value or lack thereof of treatments intended to prevent or delay the onset of maladaptive correlates of aging in human beings. This journal is indexed on PubMed Central, MedLine,

\section{Dovepress}

CAS, Scopus and the Elsevier Bibliographic databases. The manuscript management system is completely online and includes a very quick and fair peer-review system, which is all easy to use. Visit http://www.dovepress. com/testimonials.php to read real quotes from published authors. 